\title{
Dual Bioelectrical Impedance Analysis Correlates Well with Computed Tomography to Estimate Visceral Fat in Grade I-II Obese Mexican Patients
}

\author{
Ernesto G. Cardona Muñoz ${ }^{1}$, Fernando Grover Páez $^{2}$, Carlos Gerardo Ramos \\ Becerra $^{3}$, Sylvia E. Totsuka Sutto ${ }^{4}$, Guillermo Adrián Alanis Sánchez, Jesús \\ Ricardo García Corral ${ }^{6}$, Catherine A. García Aguilar ${ }^{7}$, Assen Ognianov \\ Iantchoulev $^{8}$, José Rafael Villafán Bernal, Ana Leticia Milanés Barajas ${ }^{10}$ \\ ${ }^{1}$ Chief of Department of physiology, University Center for Health Sciences, University of Guadalajara, MEXICO \\ 2,3,4,5,6,7, Department of physiology, University Center for Health Sciences, University of Guadalajara, MEXICO \\ ${ }^{9,10}$ Secretaría de Salud, MEXICO
}

\begin{abstract}
Objective: To correlate visceral fat using bioelectrical impedance analysis (Dual BIA) with computed tomography (CT) in Grade I-II Obese Mexican Patients.
\end{abstract}

Research Design and Methods: We recruited 30 Mexican patients from the western part of the country (15 men and 15 women, aged 50-65 years) with class I-II obesity (body mass index [BMI] 30-39.9 Kg/m²).

To evaluate the IAFA by Dual BIA comparing with CT.The dual BIA instrument consists of a bioelectric impedance component that measures the physical size of the abdomen. The truncal impedance is measured by applying electrical currents between the upper and lower limb leads and reading the voltage from the electrodes around the abdominal circumference. The surface impedance is measured by applying and measuring the voltage from the abdominal circumference electrodes. We took computed tomography (CT) at the umbilical level and calculated the visceral fat area (VFA).BMI was calculated as body weight (in kilograms) divided by the squared height (in meters). Waist circumference (WC) was measured at the level of the umbilicus to the nearest $0.1 \mathrm{~cm}$ in the standing position at the end of expiration.

Results: We observed a strong correlation between both methods used to calculate IAFA (BIA and CT; Pearson's $\mathrm{r}^{2}=0.659, \mathrm{p}<0.001$ ). Though both groups had significantly correlating values, the female group had a clearly stronger correlation than the male group (Pearson's $r^{2}=0.667$ vs. 0.501 ). This method exhibited a high level of reproducibility and a strong correlation with the CT results.

Keywords: obesity, intra-abdominal fat area, visceral fat, dual bioelectrical impedance analysis

How to Cite: Muñoz EG, Páez FG, Becerra CG, Sutto SE, Sánchez GA, Corral JR, Aguilar CA, lantchoulev AO, Bernal JR and Barajas AL. 2014. Dual Bioelectrical Impedance Analysis Correlates Well with Computed Tomography to Estimate Visceral Fat in Grade I-II Obese Mexican Patients ABC Journal of Advanced Research, 3, 87-92. 


\section{INTRODUCTION}

Obesity and being overweight have become two of the most important modifiable cardiovascular (CV) risk factors in Mexico. Currently, $71.3 \%$ of Mexican adults fall into one of these two categories, with a slightly higher prevalence among women and the fourth and fifth decades of life (ENSANUT, 2012; Barquera, 2013). In recent years, studies have demonstrated that the human body stores fat either subcutaneously or viscerally, and that both types increase CV risk. However, visceral fat has been shown to play a much more important role in increased CV risk than its subcutaneous counterpart (Fox, 2007). Currently, computerized axial tomography is considered the "gold standard" for measuring visceral fat (Carneiro, 2010), but its uses are limited in the clinical and epidemiological fields due to instrumentation, cost, and patient exposure to harmful radiation. Intra-abdominal fat area (IAFA) is a measure that can be used to estimate abdominal adiposity and has been used to accurately estimate visceral fat (Nagai, 2010).

Due to the high rate of obesity in Mexico and the need for an accessible method of realizing epidemiological studies in order to fully evaluate the impact of different interventions and reduce $\mathrm{CV}$ risk associated with elevated abdominal adiposity, the present study validated the DUALSCAN, HDS-2000 OMRON machine. This instrument was used to measure IAFA using bioelectrical impedance analysis (Dual BIA) in the Mexican population, demonstrating its clinical practicality (Ida, 2013).

\section{Research Design and Methods}

This cross sectional study was performed according to the protocol approved by the Ethics in Research Board of the Institute of Clinical and Experimental Therapeutics, University of Guadalajara (CEI/097/2014). The patients provided written consent to participate in this study at the Institute of Clinical and Experimental Therapeutics, University of Guadalajara. We recruited 30 Mexican patients from the western part of the country (15 men and 15 women, aged 50-65 years) with class I-II obesity (body mass index [BMI] 30-39.9 Kg/ m²).

To evaluate the IAFA, we asked our patients to wear comfortable attire with cotton undergarments (with no synthetic material or metal) to a morning appointment after fasting with an adequately emptied bladder and rectum. The patients were then asked to remove their clothing, except the undergarments, and given a disposable patient gown to wear during the procedures.

\section{DuAl BIA Method and InStrumentation}

Dual BIA calculates the cross sectional area of intra-abdominal fat at the level of the umbilicus based on the electrical potentials resulting from the application of small electrical currents in the two different body compartments. The principles of IAFA determination by the dual BIA instrument were described previously (Shiga, 2007, 2009; Yoneda, 2007). Briefly, the dual BIA instrument consists of a bioelectric impedance component that measures the physical size of the abdomen. The two sets of electrodes are for limb and truncal placement. The limb electrodes consist of four clip-on electrodes placed on the wrists and ankles. The truncal electrodes are eight pairs of electrodes $6 \mathrm{~cm}$ apart longitudinally that are fixed to a belt in pairs, one each for front and back and positioned at an equal distance. The belt is adjustable, allowing the electrodes to be centered on the mid-sagittal line at the level of the umbilicus in the supine position. The truncal impedance is measured by applying electrical currents between the upper and lower limb leads and reading the voltage from the electrodes around the abdominal circumference. The surface impedance is 
measured by applying and measuring the voltage from the abdominal circumference electrodes. Other details of the study method were described previously (Ida, 2013).

We performed computed tomography (CT) at the umbilical level and calculated the visceral fat area (VFA) using the image analysis software SINGO CT 2006A (Siemens, Somaton Emotion). The VFA demonstrated a strong correlation with the total volume of visceral fat (Ryo, 2014). We found a strong correlation (Pearson's $\mathrm{r}^{2}=0.987, \mathrm{p}<0.001$ ) between the first and second measurements taken within a month of one another(data not shown).

BMI was calculated as body weight (in kilograms) divided by the squared height (in meters). Waist circumference (WC) was measured at the level of the umbilicus to the nearest $0.1 \mathrm{~cm}$ in the standing position at the end of expiration while breathing gently at the time of Dual BIA measurement.

\section{Statistical Analysis}

All values were expressed as an average \pm the standard deviation (SD). The correlation between visceral fat areas obtained with dual BIA and CT was analyzed using Pearson's correlation coefficient. The Bland-Altman method was used to examine the mean difference and 1.96 SD between IAFA observed by Dual BIA and that estimated by CT (Bland, 1986). We calculated the sensitivity and specificity at VFA $\geq 100 \mathrm{~cm}^{2}$ using the regression equation (Oda, 2002). All p-values were two-tailed, and $\mathrm{p}<0.05$ was accepted as significant.

\section{RESULTS}

A total of 30 patients (15 men and 15 women) were included in this study, but four were eliminated from each group for not meeting the necessary selection criteria. Thus, after the selection process, 22 patients (11 per group) were eligible for VFA analysis. The average age was $54.4 \pm 4.3$ years with a BMI of $32.47 \pm 3.4 \mathrm{~kg} / \mathrm{m}^{2}$ and a waist circumference (WC) of $102.4 \pm 10.6 \mathrm{~cm}$.For the measurement of VFA by bioelectric impedance (BI), two consecutive measurements were made within a 5-minute interval (IB1 $=126.4 \pm 35.5 \mathrm{~cm}^{2} \mathrm{vs}$. IB2 $=127.3 \pm 36.6 \mathrm{~cm}^{2}$ ). The reproducibility can be measured by correlating the values through Pearson's r ${ }^{2}$ in (Figure 1).

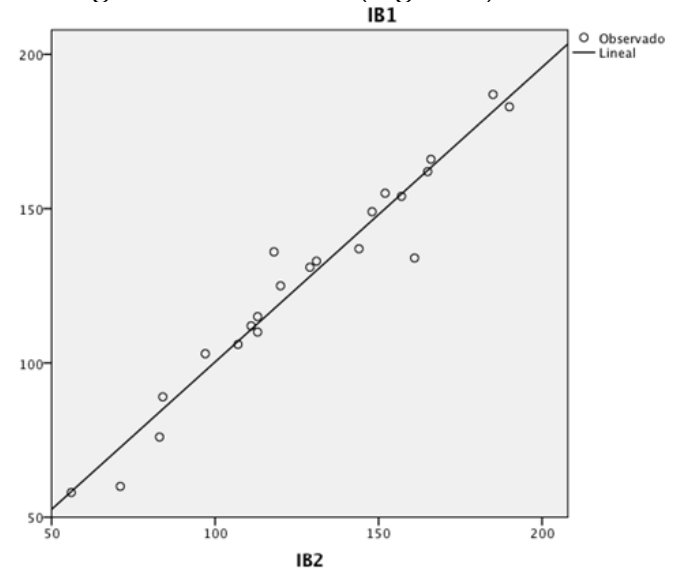

Figure 1: IB1= intra-abdominal fat area by bioimpedance analysis, first measurement vs. IB2= intra-abdominal fat area by bioimpedance analysis, second measurement $r^{2}$ Pearson $=0.982, p<0.001$

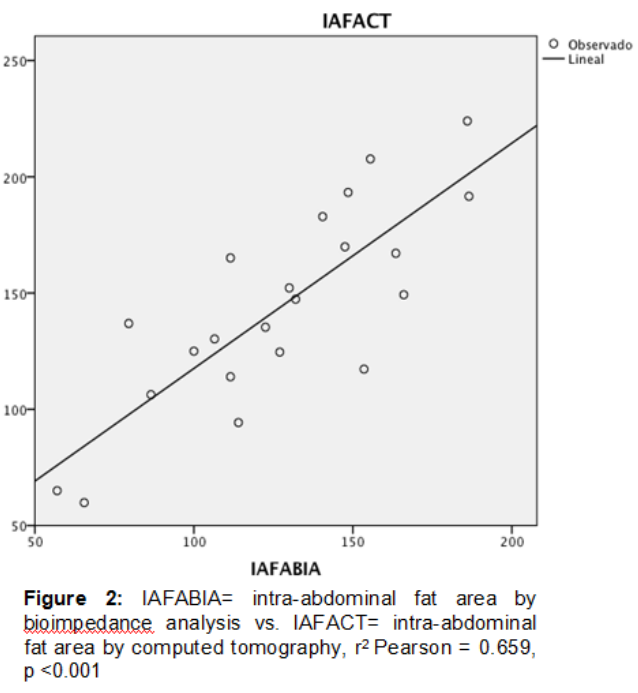


In stage 2, the average VFA values of the 22 patients obtained via (IAFABIA $=126.8 \pm 35.7$ $\mathrm{cm}^{2}$ ) were contrasted against CT measurements obtained using a planimetric estimation of Hounsfield Units $(\mathrm{HU})\left(\mathrm{CT}=143.5 \pm 42.7 \mathrm{~cm}^{2}\right)$ (Figure 2).

Results were analyzed based on gender.The average characteristics of the female group $(\mathrm{n}=11)$ were as follows: age $54.7 \pm 4.8$ years, BMI $32.3 \pm 2.8 \mathrm{Kg} / \mathrm{m}^{2}, \mathrm{WC} 107.3 \pm 2.8 \mathrm{~cm}, \mathrm{VFA}$ by BI $142.3 \pm 22.0 \mathrm{~cm}^{2}$, VFA by CT $161.9 \pm 35.7 \mathrm{~cm}^{2}$. When comparing the VFA values obtained by the two different methods, we found a significant correlation (Figure 3).

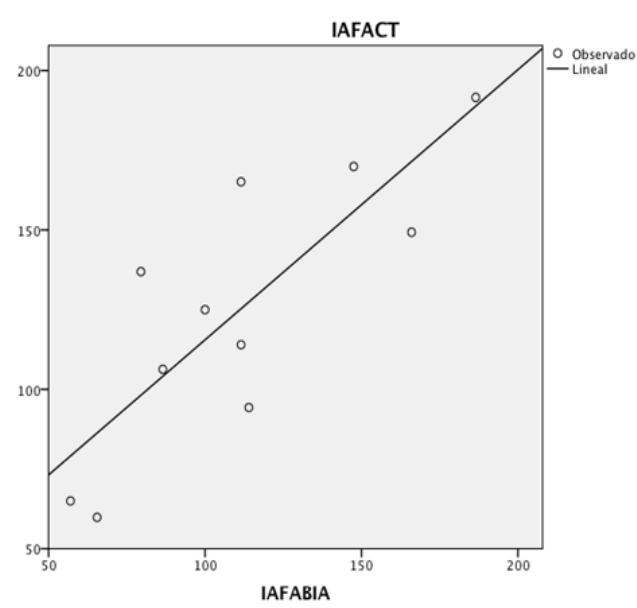

Figure 3: $\mid \mathrm{AFABIA}=$ intra-abdominal fat area by bioimpedance analysis vs. IAFACT $=$ intra-abdominal fat area by computed tomography, $r 2$ Pearson $=0.667, p=$ 0.002 .

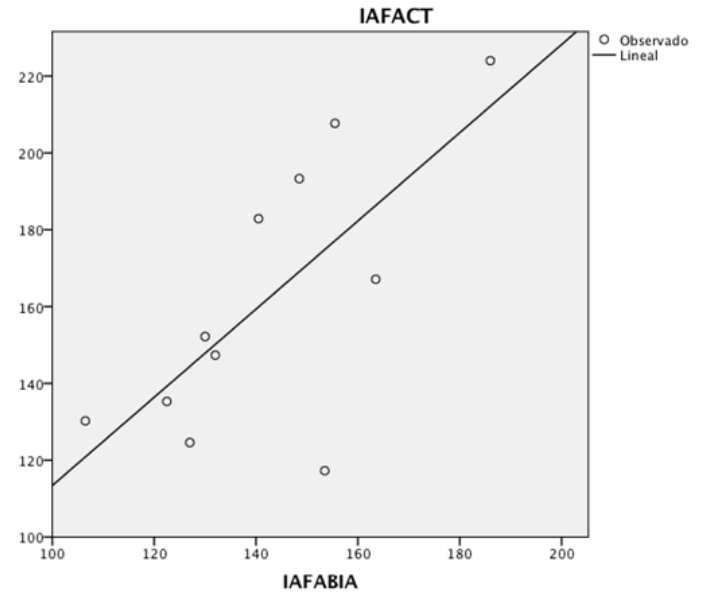

Figure 4: $|A F A B| A=$ intra-abdominal fat area by bioimpedance analysis vs. IAFACT = intra-abdominal fat area by computed tomography, $\mathrm{r} 2$ Pearson $=0.501, \mathrm{p}=$ 0.015 .

The average characteristics of the male group $(n=11)$ were as follows: age $54.4 \pm 4.3$ years, BMI $32.4 \pm 3.4 \mathrm{Kg} / \mathrm{m}^{2}$, WC $102.4 \pm 10.5 \mathrm{~cm}$, VFA by BI=126.8 $\pm 35.7 \mathrm{~cm}^{2}$, VFA by CT = 143.5 $\pm 42.7 \mathrm{~cm}^{2}$. Similar to the female group, when comparing the values obtained for the male group, we found a significant correlation between the two methods for calculating VFA (Figure 4).

\section{Discussion}

In this study we demonstrated that IAFA estimates obtained using dual BIA with the DUALSCAN, HDS-2000, OMRON instrument have high reproducibility (Pearson's $\mathrm{r}^{2}=$ $0.982, \mathrm{p}<0.001)$. We observed a strong correlation between both methods used to calculate IAFA (BIA and CT; Pearson's $\mathrm{r}^{2}=0.659, \mathrm{p}<0.001$ ). This data is consistent with the literature; Nagai (2010) also demonstrated a strong correlation between tetra polar multifrequency bioelectric impedance analysis and CT for estimating VFA $(r=0.904, p<0.01)$. On the other hand, when the group data was analyzed based on gender, we observed that the correlation persists when comparing both methods of measurement. Though both groups had significantly correlating values, the female group had a clearly stronger correlation than the male group (Pearson's $r^{2}=0.667$ vs. 0.501). This phenomenon could possibly be explained by the diminished interference in the measurement of subcutaneous fat when the electrodes are placed on the abdomen (Scharfetter, 2001). Notably, although no large difference was found in average age and BMI between the groups, the IAFA values estimated via dual BIA had greater significance in the male group, which corroborates the idea that an increase in visceral fat is a good indicator of abdominal adiposity (Cornier, 2011). On the other hand, large collaborative studies using BMI to 
define obesity have shown that the mortality risk associated with obesity markedly declines with age (Whitlock, 2009; Berrington, 2010). For this study we decided to include patients $<65$ years due to the fact that visceral fat stops being the sole predicting factor of CV risk, independent of CV mortality, starting from 65 years of age (Thomas, 2013). In contrast, other authors have shown that the risk persists in even the highest age categories when using WC to define obesity (Jacobs, 2010; Pischon, 2008). These discrepant results may be explained by the limitations of the criteria used to define obesity in clinical practice. BMI is based on body weight and height only and does not directly reflect adiposity. WC is strongly related to central adiposity (Janseen, 2002) and is associated with CV risk factors, such as hypertension, dyslipidemia, and glucose intolerance (Eckel, 2010). We found a significant correlation between WC and IAFA estimated by both methods (BI: $\left.\mathrm{r}^{2}=0.704, \mathrm{p}<0.001 ; \mathrm{CT}: \mathrm{r}^{2}=0.637, \mathrm{p}=0.001\right)$.

Our study had various limitations. Firstly, the measurement of IAFA via dual BIA and CT was not done consecutively, and included postponing the second measurement for periods of up to 24 hours for logistical reasons. This factor should be considered in future studies due to the impact it could have on outcomes as various factors may change with time, such as the state of hydration, quantity of abdominal gas, and residual urine in the bladder. The small population of patients could also be considered a possible limitation of this study due to the fact that, as the number of patients increases, the distribution curve tends to normalize, which translates into a higher level of reliability.

\section{CONCLUSIONS}

This is the first study of its kind performed on Mexican patients with class I-II obesity using the DUALSCAN, HDS-2000, OMRON instrument to estimate IAFA via dual BIA. This method exhibited a high level of reproducibility and a strong correlation with the CT results. Thus, we demonstrated the instrument's reliability, safety, operability, and cost effectiveness compared to conventional CT or magnetic resonance imaging.

\section{ACKNOWLEDGEMENT}

We thank all of the volunteers who took part in the study, and OMROM de México S.A. de C.V. for use of the dual BIA instrument.

\section{CONFLiCt INTERESTS}

The authors have no conflicts of interest to report.

\section{REFERENCES}

Barquera, S. Campos-Nonato, I. Hernández-Barrera, L. Pedroza-Tobías, A. Rivera-Dommarco, JA. (2013) Prevalencia de obesidad en adultosmexicanos, ENSANUT 2012.SaludPublica Mex. 55 supl 2. p. S151-S160.

Berrington de González, A. Hartge, P. Cerhan, JR. et al. (2010) Body-mass index and mortality among 1.46 million white adults. N Engl J Med. 363 (23). p. 2211-2219.

Bland JM, Altman DG.Statistical methods for assessing agreement between two methods of clinical measurement. Lancet 1986;1:307-310

Carneiro, AK. Lima, A. Fontes, J. Cajuhy, F. Barreto, JM. Ramos, L. (2010) Evaluaciónporimagen del Área de Grasa Visceral y susCorrelaciones con AlteracionesMetabólicas. Arq. Bras. deCardiol. 95 (6). p. 698-704.

Cornier, MA.Després, JP. Davis, N. et al. (2011) A scientific statement from the American Heart 
Association. Circulation. 124. p. 1996-2019.

Eckel, RH. Alberti, KG. Grundy, SM. Zimmet, PZ. (2010) The metabolic syndrome. Lancet. 375 (9710). p. 181-183.

Fox, CS. Massaro, JM. Hoffmann, U. Pou, KM. Maurovich-Horvat, P. Liu, C. Vasan, RS. Murabito, JM. Meigs, JB. Cupples, LA. D'Agostino, RB. Sr and O'Donnell, CJ. (2007) Abdominal Visceral and Subcutaneous Adipose Tissue Compartments: Association With Metabolic Risk Factors in the Framingham Heart Study. Circulation. 116. p. 39-48.

Ida, M. Hirata, M. Odori, S. Mori, E. Kondo, E. Fujikura, J. Kusakabe, T. Ebihara, K. Hosoda, K. and Nakao, K. (2013) Early Changes of Abdominal Adiposity Detected with Weekly Dual Bioelectrical Impedance Analysis during Calorie Restriction. Obesity. 21. p. E350-E353.

Jacobs, EJ. Newton, CC. Wang, Y. et al. (2010) Waist circumference and all- cause mortality in a large US cohort. Arch Intern Med. 170 (15). p. 1293-1301.

Janssen, I. Heymsfield, SB. Allison, DB.Kotler, DP. Ross, R. (2002) Body mass index and waist circumference independently contribute to the prediction of nonabdominal, abdominal subcutaneous, and visceral fat. Am J ClinNutr. 75 (4). p. 683-688.

Nagai, M. Komiya, H. Mori, Y. Ohta, T. Kasahara, Y. Ikeda, Y. (2010) Estimating visceral fat area by multifrequency bioelectrical impedance. Diabetes Care. 33 (5). p. 1077-9.

Oda, E. (2002) New criteria for 'obesity disease' in Japan.Circ J. 70. p.150.

Pischon, T. Boeing, H. Hoffmann, K. et al. (2008) General and abdominal adiposity and risk of death in Europe.N Engl J Med. 359 (20). p. 2105-2120.

Ryo, M. Kishida, K. Nakamura, T. Yoshizumi, T. Funahashi, T. Shimomura, I. (2014) Clinical significance of visceral adiposity assessed by computed tomography: A Japanese perspective. World J Radiol.6 (7). p. 409-416.

Scharfetter, H. Schlager, T. Stollberger, R. Felsberger, R. Hutten, H. Hinghofer-Szalkay, H. (2001) Assessing abdominal fatness with local bioimpedance analysis: basics and experimental findings. Int J ObesRelatMetabDisord. 25. p. 502-511.

Shiga, T. Hamaguchi, T. Oshima, Y. et al. (2009) A new simple measurement system of visceral fat accumulation by bioelectrical impedance analysis. In: IFMBE Proceedings. World Congress on Medical Physics and Biomedical Engineering, D€oossel, O. et al., eds., Springer-Verlag. 25/7. p. 338-341.

Shiga, T. Oshima, Y. Kanai, H. Hirata, M. Hosoda, K. Nakao, K. (2007) A simple measurement method of visceral fat accumulation by bioelectrical impedance analysis. In: IFMBE Proceedings. 13th International Conference on Electrical Bioimpedance and the 8th Conference on Electrical Impedance Tomography.Scharfetter, H. et al., eds., Springer-Verlag. 17/14. p. 687-690. Available at: http:/ /link.springer. com/chapter/10.1007\%2F978-3-540-73841-1_177?LI1/4true.

Thomas, F. Pannier, B. Benetos, A. Vischer, UM. (2013) Visceral obesity is not an independent risk factor of mortality in subjects over 65 years. Vascular Health and Risk Management. 9. p. 739-745.

Whitlock, G. Lewington, S. Sherliker, P. et al. (2009) Prospective Studies Collaboration. Body-mass index and cause-specific mortality in 900000 adults: collaborative analyses of 57 prospective studies. Lancet. 373 (9669). p. 1083-1096.

Yoneda, M. Tasaki, H. Tsuchiya, N. et al. (2007) A study of bioelectrical impedance analysis methods for practical visceral fat estimation. In: IEEE International Conference on Granular Computing. Lin, TY. et al., eds., IEEE Computer Society Press. p. 622-627. 